\title{
Hautveränderungen bei Essstörungen
}

\section{Peter Itin}

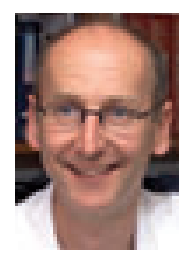

\begin{abstract}
Eine regelmässige und vielseitige Ernährung ist notwendig für Gesundheit, Lebensenergie und Lebensfreude. Eine gesunde Nahrungsaufnahme ist rhythmisch in den Tagesablauf eingebaut und gehorcht physiologischen und sozialpsychologischen Anforderungen. Bei normalem Essverhalten wird die Nahrungsaufnahme vorwiegend durch den Hunger und den Sättigungsmechanismus gesteuert. Gerät dieser Regelkreis ausser Kontrolle, kann es zu einer qualitativen oder quantitativen Fehlernährung kommen, die ebenso zu Nährstoffdefiziten führt wie Malabsorptionssyndrome. Länger dauernde Ernährungsstörungen wirken sich auf den Gesamtorganismus aus und führen somit auch zu Hautveränderungen (1). Dies gilt in hohem Masse auch für Essstörungen, die nicht als Ernährungsstörung definiert werden, sondern vielmehr Ausdruck einer vielschichtigen psychosomatischen Störung sind.
\end{abstract}

Ein gesunder Mensch, der sich normal ernährt, weist ein Körpergewicht auf, das in einem klaren Verhältnis zur Körpergrösse steht. Als Referenzwert für das Normalgewicht gibt die WHO unabhängig von Geschlecht und Alter einen Body-Mass-Index (Körpermasse in kg dividiert durch das Quadrat der Körpergrösse in Meter) von 20 bis 25 an (2).

Unter dem Begriff Essstörungen verstehen wir insbesondere die anfallsweise auftretende Esssucht ohne anschliessendes selbstinduziertes Erbrechen (BingeEating-Störung), die selteneren Anorexia-nervosa-Zustände und die Bulimia nervosa. Die genannten Essstörungen sind stets ein Spiegel schwerer psychischer Probleme, die sich durch ein verändertes Essverhalten manifestieren. Essstörungen sind so alt wie die Menschheit - in den industrialisierten Ländern haben sie allerdings in den letzten Jahrzehnten erheblich zugenommen, wobei vor allem junge Frauen betroffen sind. Hinter die- sem Phänomen stehen nicht selten Angstzustände, Zwangsgedanken, Minderwertigkeitsgefühle, eine gestörte Mutter-Tochter-Beziehung und Suizidalität. In der Regel braucht es eine geneti-

Essverhalten (2). Quantitative Essstörungen führen einerseits zu Marasmus, also zu einer Reduktion des Körpergewichts auf unter 60 Prozent der Norm, andererseits zu Adipositas, die durch einen BodyMass-Index (BMI) von $\geq 30$ $\left(\mathrm{kg} / \mathrm{m}^{2}\right)$ definiert ist. Daneben unterscheiden wir qualitative Essstörungen, die beispielsweise zu Kwaschiorkor durch Hypo-

sche Prädisposition, damit es überhaupt zu Essstörungen kommen kann. Mitverursacher sind aber auch gesellschaftliche Einflüsse und/oder Schönheitsideale, die häufig im Zusammenhang mit familiären Konfliktsituationen zu einer Veränderung des Körperschemas und damit zu einer Störung der Körperempfindung führen. Diese komplexe Konstellation resultiert schliesslich in einem veränderten Essverhalten, das bei Anorexie zu Gewichtsverlust führt und beim Binge-EatingPhänomen in Fettleibigkeit enden wird. Die körperlichen Folgezustände haben ihrerseits wieder Rückwirkungen auf das proteinämie führen oder durch besondere Diäten induziert werden, die mit einem Vitamin- und Spurenelementmangel einhergehen können.

\section{Binge-Eating-Syndrom}

Beim Binge-Eating-Syndrom besteht ein zeitweiser Kontrollverlust des Essverhaltens, der sich in regelmässigen Fressattacken manifestiert, ohne Bedürfnis nach selbstinduziertem Erbrechen. Meist sind komplexe seelische Konflikte auslösend. Das Lebensrisiko in der westlichen Bevölkerung beträgt 3,5 Prozent. Eine solche Essattacke ist definiert dadurch, dass 


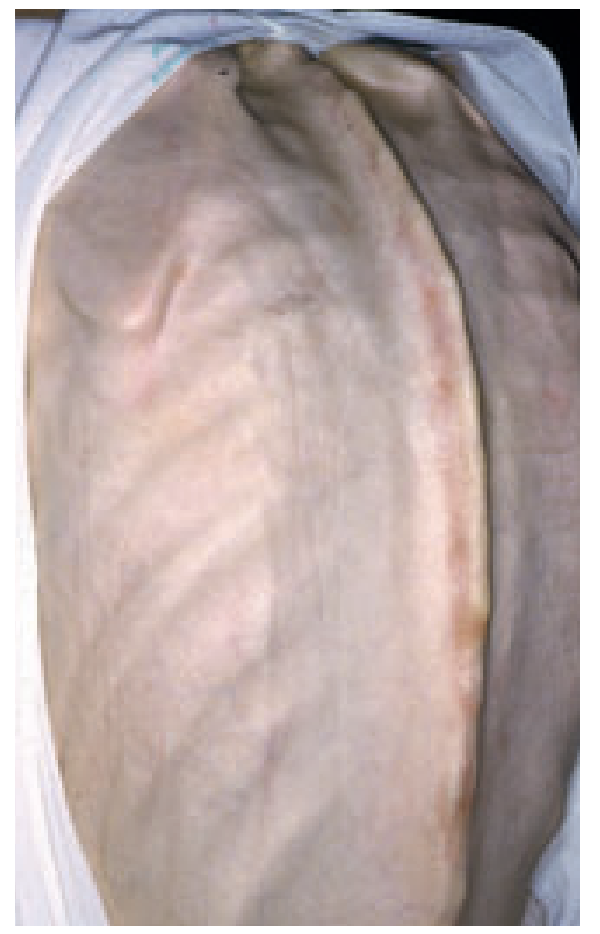

Abbildung 1: Anorexie mit Kachexie

innerhalb von Minuten bis zu zwei Stunden ungewöhnlich grosse Mengen an Nahrungsmitteln konsumiert werden, die vorwiegend reich an Kohlenhydraten und Fetten sind, jedoch arm an Vitaminen und Mineralstoffen. Aus diesem Grund können langfristig Mangelerscheinungen auftreten. Betroffene sind nicht mehr in der Lage zu kontrollieren, wie viel sie essen oder wann sie mit dem Essen auf-

Tabelle: Hautveränderungen bei
Patientinnen mit Anorexia
nervosa/Bulimie in absteigender
Häufigkeit
- Trockene Haut
- Faziale Purpura nach Erbrechen
- Cheilitis
- Hypertrichose
- Diffuses Effluvium/Alopezie
- Akrozyanose
- Russell-Zeichen
- Gingivitis/Aphthen
- Nagelveränderungen
- Hungerödeme
- Trichotillomanie
- Artefakte
- Parotisschwellung
- Aurantiasis (Hyperkarotenämie)
Adaptiert nach (3)

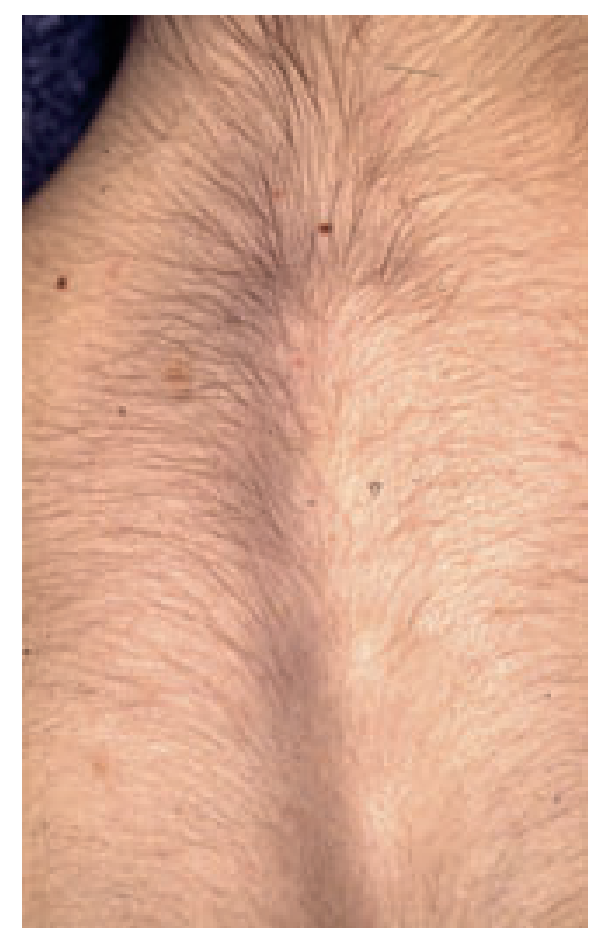

Abbildung 2: Anorexie mit generalisierter Hypertrichose

hören müssen - die meisten sind, beziehungsweise werden daher übergewichtig. Das Binge-Eating-Syndrom wurde erstmals 1959 als solches geprägt, und die eigenständige Diagnose ist in den USA erst seit 1994 akzeptiert.

\section{Anorexia nervosa:}

Die Anorexia nervosa ist eine verbreitete Essstörung bei weiblichen Jugendlichen und jungen Frauen und ist meist Ausdruck erheblicher psychischer Konflikte. Die Betroffenen sind davon überzeugt, dass sie mit einem Körper, der dem heutigen Schönheitsideal entspräche, von ihrer Umgebung mehr Liebe und Anerkennung erwarten könnten. Seelisch sind sie am Verhungern und leben dies körperlich aus. Heute sind bereits 35 Prozent der an Magersucht erkrankten Patienten Männer - deren Zahl nimmt stetig zu. Die Chance, im Laufe des Lebens eine Anorexie zu entwickeln, beträgt für die westliche Gesamtpopulation 0,9 Prozent. Die Anorexia nervosa ist charakterisiert durch bewusste Verweigerung genügender Kalorienaufnahme, was zur Kachexie sowie zu metabolischen und endokrinologischen Veränderungen führt, die lebensbedrohlich sein können. Die Diagnose

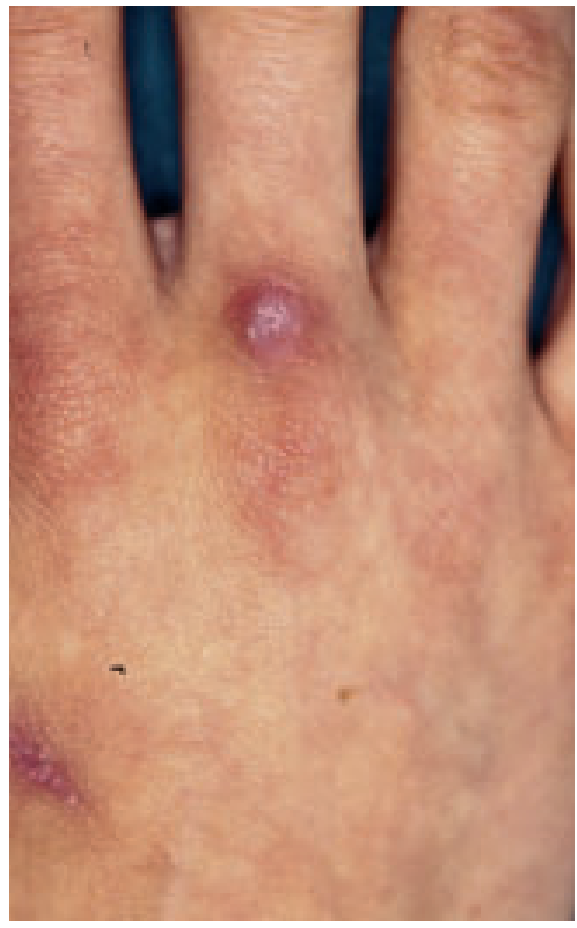

Abbildung 3: Russell-Zeichen bei Bulimie

wird erst erstaunlich spät gestellt und die Behandlung somit stark verzögert begonnen.

\section{Charakteristische Hautverände- rungen bei magersüchtigen Patientinnen}

Zur Identifizierung charakteristischer dermatologischer Marker, die eine für die Prognose entscheidende Früherkennung der Anorexia nervosa erlauben würden, untersuchten wir 21 magersüchtige junge Patientinnen (im Alter von 19 bis 24 Jahren) in einem retrospektiven und einem prospektiven Studienanteil (2). 7 von ihnen wiesen eine zusätzliche $\mathrm{Bu}$ limie auf. Als Kontrollgruppe dienten 7 gesunde weibliche Jugendliche derselben Altersgruppe. Im Vergleich zur Kontrollgruppe (29\%) wiesen 71 Prozent der Anorektikerinnen eine deutliche Xerodermie auf, 76 Prozent eine Cheilitis, bei 62 Prozent fiel eine allgemein verstärkte Körperbehaarung auf. Bei 67 Prozent der Anorexie/Bulimie-Patientinnen liessen sich hyperkeratotische Läsionen über den Fingergrundgelenken beobachten (sog. Russell-Zeichen), die durch selbstinduziertes Erbrechen ausgelöst werden. Weitere auffällige Befunde waren trockenes 


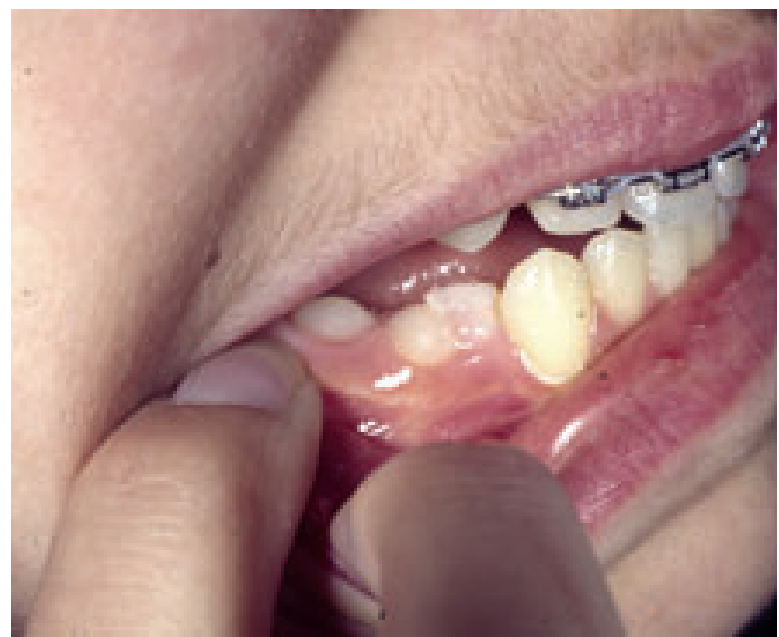

Abbildung 4: Zahnschmelzerosionen bei Bulimie

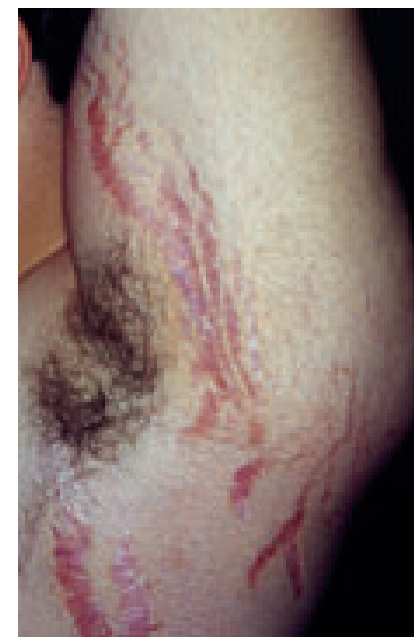

Abbildung 5: Striae distensae

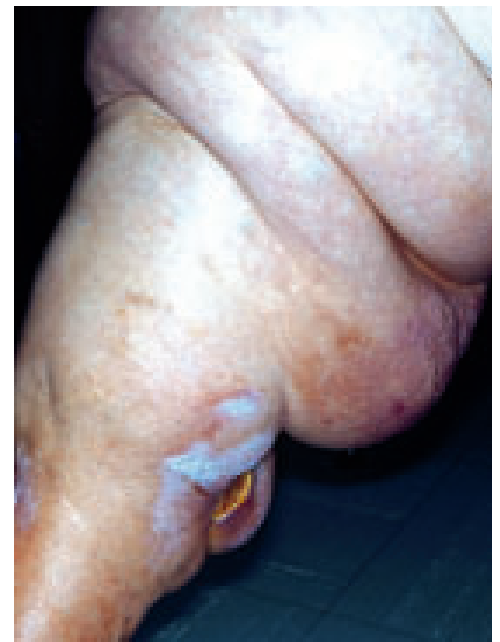

Abbildung 6: Ulcus cruris bei Adipositas
Kopfhaar und Periungualerytheme (je 48\%), kalte Akren (38\%), Akrozyanose (33\%), Zahnfleischveränderungen (37\%) und Nagelveränderungen (29\%). Die Studie dokumentiert erstmals, dass ein BMI $\leq 16\left(\mathrm{~kg} / \mathrm{m}^{2}\right)$ als kritischer Wert betrachtet werden muss, da hier vermehrt dermatologische Veränderungen zu beobachten sind. Die Kenntnis der geschilderten und für die Erkrankung typischen Hautbefunde erleichtern es Dermatologen und Hausärzten, eine vorliegende Anorexia nervosa frühzeitig einer angemessenen Therapie zuzuführen, was die Prognose wiederum signifikant verbessert.

Ein Teil der Patientinnen mit Anorexia nervosa weist zudem eine ausgeprägte Hyperkarotinämie auf, bedingt durch die exzessive Aufnahme karotinreicher Gemüsesorten, die, obwohl kalorienarm, dennoch ein Völlegefühl vermitteln können. Darüber hinaus zeigen anorektische Patienten häufig auch eine Trichotillomanie; 30 Prozent dieser Patienten weisen eine Trichophagie auf, 1 Prozent hat eine persistierende Trichophagie, sodass die Gefahr eines sogenannten Trichobezoar mit abdominaler Raumforderung besteht (Rapunzel-Syndrom). Im Rahmen einer ungewöhnlichen Essstörung haben Grimalt und Happle die Trichorhizophagie beschrieben, bei der eine betroffene $\mathrm{Pa}$ tientin lediglich die Haarwurzeln kontinuierlich gegessen hat [3]. In unserer Studie hatten 3 von 21 Patienten eine Trichotillomanie.

\section{Bulimia nervosa:}

Bei der Bulimia nervosa handelt es sich um eine Essstörung mit attackenweiser, übermässiger Nahrungsaufnahme, mit nachfolgendem selbstinduziertem Erbrechen. 1,5 Prozent der Bevölkerung wird eine Bulimie erleiden. Manchmal ist die Bulimia nervosa kombiniert mit einer Anorexia nervosa. Sichtbare Markerveränderungen bei der Bulimie sind eine ausgeprägte Speicheldrüsenvergrösserung, durch Magensäure induzierte Zahnschmelzdefekte wegen des rezidivierenden Erbrechens, eine anguläre Stomatitis, Gingivitis, Perlèche und das sogenannte Russell-Zeichen. Manchmal fallen auch pharyngeale Würghämatome auf als Phänomen eines wiederholten selbstinduzierten Erbrechens. Patienten mit einer Anorexie weisen häufiger als Kontrollkollektive eine Thrombopenie auf und leiden unter Vitamin-K-Mangel, der ebenfalls mit einer erhöhten Blutungstendenz assoziiert ist.

\section{Adipositas:}

Während Übergewicht durch einen BMI von > 25 definiert wird, spricht man bei BMI-Werten > 30 von Fettleibigkeit (Adipositas). 30 Prozent der Amerikaner, 32 Prozent der Brasilianer, 7 Prozent der Franzosen und 4,3 bis 17 Prozent der Chinesen leiden an einer Adipositas. Die übermässige Gewichtszunahme führt zu zahlreichen dermatologischen Folgeerscheinungen wie Striae distensae,
Intertrigo, plantare Hyperkeratosen, Pseudoacanthosis nigricans, Skin tags, Hyperhidrose und im Extremfall zu Ulcus cruris und Lipodermatosklerose (Abbildung 5 und 6) (4).

Zusammenfassend lässt sich sagen, dass Essstörungen eine Zeiterscheinung darstellen und in der industrialisierten Welt eine besonders hohe Prävalenz aufweisen. Sie sind Ausdruck komplexer psychischer Konfliktsituationen und müssen ernst genommen werden. Es gibt zahlreiche Hautveränderungen, die auf das Vorliegen von Essstörungen hinweisen und eine frühzeitige Diagnose ermöglichen (5).

\section{Korrespondenzadresse:}

Prof. Dr. Peter Itin

Chefarzt Dermatologie

Universitätsspital Basel

Petersgraben 4, 4031 Basel

\section{Literatur}

1. MacDonald A, Forsyth A: Nutritional deficiencies and the skin. Clin Exp Dermatol 2005; 30: 388-390.

2. Hediger C, Rost B, Itin P: Cutaneous manifestations

in anorexia nervosa. Schweiz Med Wochenschr 2000; 130: 565-575.

3. Grimalt R, Happle R: Trichorhizophagia. Eur J Dermatol 2004;14: 266-267.

4. Scheinfeld NS: Obesity and dermatology. Clin Dermatol 2004; 22: 303-309.

5. Strumia R: Dermatologic signs in patients with eating disorders. Am J Clin Dermatol 2005; 6: 165-173. 